\title{
Potential of Musical and Pedagogical Analysis of Socio-cultural Interaction Processes
}

\author{
Anna Iosifovna Scherbakova ${ }^{1}$, Alexander Vladlenovich Kamenets ${ }^{1} \&$ Elizaveta Olegovna Zinchenko ${ }^{1}$ \\ ${ }^{1}$ Russian State Social University, 1, 4, Vilgelma Pika st., Moscow, Russian Federation \\ Correspondence: Anna Iosifovna Scherbakova, Russian State Social University, 1, 4, Vilgelma Pika st., Moscow \\ 129226, Russian Federation. E-mail: anna.68@list.ru
}

\author{
Received: August 17, 2014 Accepted: August 30, 2014 Online Published: November 27, 2014 \\ doi:10.5539/ass.v10n24p144 URL: http://dx.doi.org/10.5539/ass.v10n24p144
}

\begin{abstract}
The article considers innovative approaches to the study of social interaction on the basis of the use of techniques of the musical forms analysis. As the baseline for revealing these approaches the pedagogical process in teaching music disciplines is elucidated. The authors reveal some algorithms of such analysis opening up new perspectives not only in music pedagogics but also in the study of cultural processes: their structure, internal logic, and dynamics of development. The analysis, demonstrated in the article, represents essentially new ground for researches for music pedagogics, cultural science and social anthropology delimited from the minor impacts and factors in the study of cultural processes.
\end{abstract}

Keywords: interaction, cultural process, modeling, forecasting, consolidation, musical form, intonation, synergetic, cultural dynamics, frame, dissonance, conflict

\section{Introduction}

One of the pressing problems in culturological and pedagogical researches is the non-study of the social interaction processes in different spheres of human practice: in industrial, public, pedagogical and other spheres of human activities. The existence of this problem is caused by two reasons of research character. The first reason is the non-development of the conceptual and terminological apparatus, of the corresponding model and conceptual ideas, of real social and cultural processes adequate to phenomenology of dynamics, as the prevailing morphological and functional approach to the analysis of these processes can provide only the "elder" conditions of social systems and cultures. Designed by researchers (culturologists, cultural anthropologists, teachers, sociologists, ethnographers, etc.) dynamics of development of societies, cultures, civilizations, social groups, etc. has mainly speculative and hypothetical character, beginning from the theories of "passionarity" of development of civilizations and ending with different evolutionistic theories and doctrines of societies, development of a personality, etc. (Asafyev, 1971; Zinchenko, 2014, p. 110; Kamenets, 2012; Kamenets \& Seleznyova, 2013).

This statement has objective character in many respects as no process can be studied in statics and dynamics simultaneously. G. P. Schedrovitsky wrote about this methodological difficulty on the example of the analysis of human activities as a process: "Constant transformation of "successive", i. e. deployed and proceeding in time, process, to "simultaneous", i.e. what is happening in full its structure in a moment, is a fact that had long been fixed in different studies of human mental activity. He made researchers think that in so-called successive process at each moment of time not all the structure of the studied whole but only part of it, and at different moments of time-functionally different parts-is implemented. The activity, taken in its minimum objective integrity, appeared as "blurred" in time: its different parts and elements are implemented at different times and yet there are such relationships and dependencies between them that (thanks to some specific mechanisms) work all this time and unite all the elements in one integral structure that was not in the processes of change of elementary objects." (Schedrovitsky, 1995, p. 240). Impossibility of identification of integral structure of processes on the basis of the existing descriptions of any human activity led G. P. Schedrovitsky to an unfavourable conclusion: "on the basis of the category "process" it was never possible to explain how a person acts, how he uses his last products as means of new activity, how he unites "past", "present" and "future" in one actual structure. (Schedrovitsky, 1995, p. 240). The situation has not changed by now. There is still an unsoluble problem of the study of full structure of these processes for researchers of social and cultural processes, as the established research procedures and appropriate analysis techniques tend to study their static but not dynamic, 
ever-changing structures, including in pedagogical activity.

\section{Methods}

Methods describe in detail how a research passed, what problems were touched upon, a variant of way out of a problem situation is offered. Humanitarian knowledge has a plenty of methods used in various researches, related to a person, society, culture. The authors of the article chose the methods based on introduction of a system of interaction of musical forms to educational and upbringing process. The situations of a learner's education and motivation for knowledge acquisition are considered on the examples of interaction between a teacher and a learner.

\subsection{Problem Description}

The described above situation results in the met insolvency of socio-cultural and pedagogical planning and forecasting very often "lagging behind" dynamics of social and cultural processes and in their inadequate estimate by all subjects of social and cultural society life.

Another reason of the insufficient study of the social interaction processes, important for any human activity, consists in explicit or implicit substitution of interaction processes by most of researchers for processes of socio-cultural and pedagogical action, activities, impacts in which mainly unilateral activities of subjects, actors of these activities without considering peculiarities of all participants of social and pedagogical processes are anyway studied and described (Chen et al., 2012). In practice it leads to impossibility of the effective solution of many social, cultural and pedagogical tasks vital for society both at the micro level and at the macro level (for example, making social and cultural projects considering all the participants' possibilities of interaction, formation of civil society representing interests of all social groups, formation of the state cultural policy based on various interaction of most of members of society as active participants of cultural processes, on the inefficiency of many institutional pedagogical practices, etc.).

The situation is not saved by achievements of symbolic interactionism (in psychology, sociology, cultural science, social and cultural anthropology) which, eventually, reduce the study of real interactions by the study of communications, communication processes, cognitive activity considerably reducing and often even leveling the very possibility of research of social and cultural interaction processes, including interacting people's behavioral and mental activities. This substitution is inevitable if in research of interaction processes one is limited to the established verbal-conceptual simultaneous description and analysis which are not "catching" dynamics of socio-cultural and pedagogical interaction processes in its integrity.

\subsection{Authors' Methods}

The authors of the article offer one of possible versions (models) of the analysis and description of the processes of socio-cultural and pedagogical interaction (teachers and learners) using as means of the analysis of a musical form adequate to this task, which is built, as a rule, in the form of a process of formation, development and completion of the interaction process of different themes, melodies, sound ratios, etc. If to take into account that musical art in one way or another reflects real social life of people, dramatic nature and peripetias of interaction of their emotional states, inclinations, aspirations (music semantics as a version of cultural semantics), reliance on such an analysis looks rather correct (Zeynep et al., 2014). "Multiplicity of meanings inherent in every musical composition, dictates the need for multiple interpretations, the ability to think dialogically comparing different concepts and approaches to understanding of the integrity of the musical text in its unity with the historical context, with the features of creative thinking of the era, author's artistic and value orientations of the studied musical composition" (Scherbakova, 2014). It is impossible also not to note in this regard that "philosophical comprehension of music and music education turns a researcher to a wide range of humanitarian knowledge inevitably: to philosophy, sociology, psychology, cultural science, pedagogics, musicology, art studies, etc." (Scherbakova, 2014).

This in turn opens up new possibilities in the field of design and forecasting of many social, cultural and pedagogical processes taking into account a phenomenon of their participants' interactions.

The logic of development of musical forms can be extrapolated to the logic of development of these or those processes of social interaction as both ones are manifestation of socio-cultural subjects' social activities, possess own intentionality and immanence (Carl et al., 2009; Villodre, 2014). The musical art is created by people and for people embodying cultural semantics in the form of images, feelings, "musical thoughts", etc. reflecting deep regularities of social relations "passed" through a personality's emotional sphere (Asafyev, 1971). Besides, the music language, appeal to musical structures allows to describe those procedural aspects of social interaction which are poorly seized by usual discrete language descriptions. The musical material itself, included in the 
process of interaction of teachers and learners, creates conditions for special psychological space in pedagogical activity. Accordingly, "one of the important mechanisms of creation of synthetically-dialogical paradigm in musical-pedagogical education is empathy" (Scherbakova, 2012, p. 115) between a teacher and learners.

In this case a synergetic social-psychological effect appears as a result of interaction between teachers and learners which can be called a bioenergy field stimulating involvement in procedurality of the corresponding partnership close to the phenomenon of a musical form. In this regard in some existing descriptions of interaction processes the first approaches to creation of special "notation" by analogy with the sphere of the musical art have been already developed by means of analytical psychology opening up new possibilities in modeling and studying socio-cultural and pedagogical processes.

Thus, describing the social interaction processes there are already sign-symbolical fixations of its existential, power and physical characteristics, their dynamics, arising "bifurcation points", conflicts and their resolutions, harmonizations, polyphonies, etc. Another important resource in substantial and structural interpretation of socio-cultural processes consists in creative using the available dictionary of musical nuances which are capable to enrich the existing cultural semantics considerably in the description of social processes.

\section{Results}

The preliminary comparisons of sociolinguistic and musical characteristics of social interaction processes on the example of the consolidation process in the musical-pedagogical situation which is of particular importance for ensuring the full pedagogical process (a teacher and a learner's relationship) under the laws of a musical form, identical to the logic of the developed studied musical contents, are offered. B. Asafyev's known work "Musical form as a process" is taken as a basis of the offered analysis (Asafyev, 1971).

\subsection{Model of a Pedagogical Situation}

The initial pedagogical situation in studying music disciplines may be called as consolidation between a teacher and a learner. This consolidation may begin with comparison of its sociolinguistic characteristic as "friendliness" in interaction with a learner by a teacher; its corresponding adequate musical characteristics (in interaction between a teacher and a learner on the corresponding musical material)-recognition in music giving pleasure; removal of a melody far away from keynote to draw a listener's attention (novelty intrigue in motion) (Baker, 2013). In the musical-pedagogical situation this process stage can be considered as spontaneous awakening of learners' interest in mastering musical material together with a teacher, where there is a transition from the recognized musical contents to the unexpected ones accompanied by a teacher's comment, interaction directed at the search of extraordinary things in the ordinary ones ("The world is nothing without astonishment", the poet M. Svetlov wrote). The emergence of the consolidation process of a teacher with learners occurs as separation from the "unfriendly" (the unfriendliness of intonations, musical contents, etc.) which is estimated as "alien" (unfriendly) towards consolidation.

\subsection{Example of Model Functioning}

An example of this model can be the comparison of beauty of harmony of classics and vulgar popular music within one theme of creativity, experience, image or within one piece of music, reading of inner meaning of friendly and unfriendly musical movements with inclusion of literary, art-graphic associations, etc. by a learner and a teacher. Thus "friendliness" and "unfriendliness" are equally important for formation of the consolidation process of a teacher with learners. The unfriendliness arises as remoteness from a consensus in this case. The "alien" (unfriendly) is necessary for tending of this process to an initial consent ("keynote"), for more obvious fixing of essence of the starting point of consent. Thus, the consolidation process is initially split into "friendliness" and "unfriendliness" from the starting point of its moving. And this split is initiated by the "friendly" participants of the process for consolidation strengthening, for giving a meaning to it ("friendliness" and consent are implemented concerning "something"-for example, concerning the steady judgment embodied in the corresponding musical thought tending to a keynote). Further this consolidation process can strengthen an element of opposition in itself, discrepancy of positions, estimates of the musical material, perception of it (the corresponding musical characteristic: dissonance). The aggravation of an ambivalent condition of the consolidation process happens, initiated by a teacher, aggravating disagreements of a teacher and learners towards the musical material to more sharply designate the need to return to the initial positive pedagogical and musicological estimates of this material (for example, carrying to the point of absurdity disagreements, a learner's musical preferences). As a result a learner himself already starts tending to this consent as he does not offer steadiness alternative to consolidation. The discording element is non-constructive in respect of alternative constructability here. It destroys to even more strengthen moving to consolidation. This is organized by a teacher "filial" learner's discontent with "paternal" consolidation process but without filial revolt. Then the consolidation 
process of a teacher and learner develops on the basis of the sociolinguistic characteristic "reliability" accompanied by the corresponding musical characteristic-approval of the main musical tone (initial teacher's statements, estimates, etc.). Reliability (fixing) of consolidation (check of its strength), its "immunostaining" for alien elements (for example, for inadequate estimates of the musical material, a teacher's actions by a learner) is provided in such a way. Next, a teacher shows the usefulness of further study of the musical material for a learner himself (the corresponding musical characteristics of this pedagogical directive are steady and useful intonations. Thus, the consolidation definiteness is maintained as through the usefulness, moving the process, and through the steadiness providing repeatability of the essential characteristics of the process reproducing the initial position of the consolidation process anyway (Kenneth et al., 2006). Next, a teacher organizes conflict with his learner (the problem of learning) which in the musical aspect can be presented as the conflict between the main and collateral parties. The pedagogical contents of this conflict consists in demonstration of a teacher's irreconcilability to behavioral, verbal or performing learner's manifestations as to still shown "remnants" of the last experience resisting to pedagogical tasks (Thompson, 2013). As both parties nevertheless maintain "peace", accepted by them in the consolidation process, a learner wittingly agrees to a role of a weaker bearer of the "collateral" party than a bearer of the "main" party (teacher). The interacting participants continue keeping, therefore, contact according to the laws of a musical form. So, the victory of the main over the "collateral" conflicter as indicative process for even greater consolidation supporting the main conflicter (teacher) is specially organized. Such specially organized aggravation of opposition of a teacher and learner will not be destructive as both parties have already accumulated rather sound experience of matches of estimates and frames (of a performer or listener) of a learner and teacher.

\subsection{Models of Using the Musical Analysis Techniques in the Pedagogical Process}

Then in the pedagogical interaction under consideration such versions of using the musical analysis techniques and design of the pedagogical process on the study of music as expansion of borders of the theme under the study (by analogy with expansion of a theme in a piece of music) are possible; this expansion is detection of new socio-cultural reality receiving legitimacy and importance through the inclusion in the main theme at the same time; comparison (mutual imposing) of already found (as in music) studying sub-themes (spheres, realities) in the name of their synthesis. Then the pedagogical process may become more complicated, polyphonize and come to an end with some result in the form of an artifact (new knowledge, performing skills) in which the previous micro processes (pedagogical result) "are compressed", concentrated. Next, such characteristic as a pedagogical action, fixing the mastered material, may be included. It can be estimated as such musical characteristic as imitation of the main theme. Thereby, within the consent paradigm the process of pedagogical interaction proceeds through the miniprocesses imitating the main theme; any deviations from the main theme must not lead to leaving it (Nakahashi, 2014). These deviations, thus, "spin" round the initial centre (the main pedagogical thesis). The musical characteristic corresponds to it as division of functions between the steady and unsteady intonations for maintenance of dynamic balance; polyfunctionality inside the musical process. Accordingly, there is combination of the steady and unsteady at continuous violation and return of the balance; avoiding absolute repetitions in moving when taking up the main theme of the process by its different subjects in the delay mode ("And the last will be the first" what is especially important for "lagging behind" learners). Next, the interaction between the subjects of the process as already equal partners (co-performers) occurs what can be estimated as musical polyphony. The interaction of the parties (subjects) in the process is carried out as synchronously (vertically, i. e. at each moment of time taking into account the given mental, social and other states of interacting participants at the given moment of time) and diachronously (horizontally, in dynamics) (Asafyev, 1971). Each of the parties conducts the line and reveals its aspect of the main theme (disposition) of the process providing integrity of the latter (integrity through a variety of the parties or micro processes). Here the rational and emotional, existential aspects of interaction on the corresponding musical material draw together as much as possible. As a total result of formation of the consolidating interactions it is possible to consider finding special sociality by learners which in the musical aspect can be estimated as mastering the principles of organization of a musical form which in real social interaction are considered as the unity of external manifestations of a personality and its internal contents, the appropriateness of the shown socio-cultural activity. As fixing of already acquired skills the change of the process of consolidation of learners with a teacher can be initiated to the learners. This process gains new quality through the repetition of structural ratios of elements of the process but already in a distance from the initial process, in unexpected spaces and in spheres (change of keys); also the process orientation changes from "+" to "-" or vice versa, also through abnegation of the process from the point of view of its result (moving to the opposite result-"change of fret"); emergence of the contrast micro processes providing the process change as an incentive for its development; also-emergence of the companions of the main process theme as a "dialogue" of the micro processes moving to one direction. 


\subsection{Field of Application of the Structural Analysis of a Piece of Music in the Pedagogical Process}

The offered structural analysis can be used as for the study of the concrete musical material with a learner and (that is especially important for us) in the methods of teaching. For example, the change of keys can be interpreted as the use of new illustrations to explain the essential contents of the musical material by a teacher; "change of fret"-as alternation of "minor" and "major" perception of cultural semantics of this material; contrast in deliberate distortion of the set "norm of perception" by a teacher to strengthen fixing and tending of learners to this norm, etc. With the same purpose change of a rhythm of the mastering process of the same musical contents is possible to mark out singularity of the mastered musical material. In musicological aspect this pedagogical action can be characterized as rhythm-intonational distinction of two or several re-interfaced elements. Repetition becomes here an incentive for moving in comprehension of a piece of music towards its uniqueness and singularity. At last, a learner is offered to reproduce the somehow acquired musical contents (in verbal or in a performing form) independently. Thereby, the process of studying music is finally institutionalized, changes towards finding the steady cultural patterns of activities as a response to the requirements of the organized musical environment (in the form of a teacher's actions and sounding of music). We will limit ourselves to comparison of the pedagogical and musical characteristics of the consolidation process of teachers with learners in mastering the musical culture.

This comparison can be carried out towards other paradigms of social interaction (conflict and partner paradigm) (Kamenets \& Urmina, 2011). And the most appropriate way of mastering the musical material here is the wide use of musical improvisation techniques and free associations by learners on the basis of the considered above technologies of the musical processes analysis. Learners in this case become to some extent co-authors of the offered pieces of music. We will also note that there are different versions of social-anthropological diagnostics now allowing to use these pedagogical strategies and musical material taking into account the results of such diagnostics according to the laws of a musical form.

\section{Discussion}

The given above comparison of the pedagogical and musical characteristics "can be estimated as one of the possible methodological strategies in modeling, design" (Kamenets, 2012, p. 69) and the study of socio-cultural and pedagogical processes to solve a variety of social, educational and pedagogical problems. The use of music-ontologic ideas in the pedagogical processes can be considered as realization of a principle of cultural congruence and aesthetic-oriented pedagogics in the process of teaching the music disciplines having impact on all learners' inner world and their social behavior (Scherbakova, 2012). In the offered research approach the preliminary general methodological directives, connected with using the musical analysis techniques in studying the cultural and pedagogical processes, have been specified. In the future the rapprochement of musical terminology, the use of laws of a musical form and culturological and also pedagogical terminology and principles of the cultural-anthropological and didactic analysis in modelling and forecasting of socio-cultural dynamics on the micro and macro level of social reality is planned (Haritatos \& Benet-Martínez, 2002). This direction is rather innovative but it was affected in A. I. Scherbakova's works, Yu. N. Kholopov's ones and other (Kholopov, 2008; Scherbakova, 2014). Also the processes of modelling and forecasting in the social and cultural sphere are quite well revealed in works on socio-cultural design where the term design is defined as an activity form possessing the signs of advancing reflection of reality during which a preimage (prototype) of an assumed object, phenomenon or process by means of specific methods is created (Zinchenko, 2014).

\section{Conclusion}

The preliminary comparison of the pedagogical and musical characteristics of socio-cultural interaction processes on the example of teaching the music disciplines shows the availability of real possibilities of overcoming of the known gap between studying music and its verbal commenting in which there is an inevitable risk of substituting learners' live perception of the musical material for teachers' schematically or tendentious commenting.

The rapprochement of the logic of development of a musical form with the pedagogical logic can also facilitate the emergence of synergetic effect in interaction of teachers and learners and also opens up new perspectives in work at musical repertoire. The latter is mastered in parallel with the logic of music semantics mounted according to the forms of a piece of music as a model of real interaction of a teacher with learners, whether it is performing repertoire or works as the material of musical-educational activity (Wright \& Lundy, 2014). Thereby, music starts carrying out the form-building function towards learners' real social behavior in the process of their contacting with music teachers. In this case in music pedagogics accents are placed a little differently in comparison with traditional musical-pedagogical activity. The study of music as a matter of fact becomes a 
reason for direct impact on learners' consciousness, attitude and behavioral directives and is subordinated to this task first of all. Accordingly, the probability of formation of musical interests and demands in the context of everyday life activities increases sharply.

Music becomes "a window to the world", powerful cognitive means in gaining life experience and steady outlook. Using the principles and technologies of the musical forms analysis is also an extremely productive strategy for actually culturological researches. The perspectives for studying the structure and dynamics of cultural processes, which (especially at the micro level) have not been studied well till now, open up. The very musical analysis techniques are capable to enrich the conceptual and terminological apparatus of cultural science considerably, first of all in studying dynamics of culture, to give greater strictness to modeling and forecasting of cultural processes what is not only of theoretical but also of great practical importance (for cultural policy, development of socio-cultural programmes, optimization of processes of inculturation and socialization) of a personality) (Nigmatov, 2014). The opening up corresponding possibilities of socio-cultural design should be especially allocated. The advantage of design technology in comparison with other methods of purposeful socio-cultural changes is that it combines the normative and diagnostic approaches, characteristic for programming and planning, as socio-cultural design "has a great potential in solving pressing social problems of society" (Zinchenko, 2014, p. 107).

\section{References}

Asafyev, B. (1971). Musical form as a process (2nd ed.). Leningrad: Music.

Baker, K. (2013). Information literacy and cultural heritage: A proposed generic model for lifelong learning. Information Literacy and Cultural Heritage, Chandos Information Professional Series, 117-133. http://dx.doi.org/10.1016/B978-1-84334-720-0.50005-2

Carl, P., Maertz, Jr., Hassanb, A., \& Magnussonc, P. (2009). When learning is not enough: A process model of expatriate adjustment as cultural cognitive dissonance reduction. Organizational Behavior and Human Decision Processes, 108(1), 66-78. http://dx.doi.org/10.1016/j.obhdp.2008.05.003

Chen, H., McAdams-Jones, D., Tay, D., \& Packer, J. (2012). The impact of service-learning on students' cultural competence. Teaching and Learning in Nursing, 7(2), 67-73. http://dx.doi.org/10.1016/j.teln.2011.11.002

Haritatos, J., \& Benet-Martínez ,V. (2002). Bicultural identities: The interface of cultural, personality, and socio-cognitive processes. Journal of Research in Personality, 36(6), 598-606. http://dx.doi.org/10.1016 /S0092-6566(02)00510-X

Kamenets, A. (2012). Musical characteristics of socio-cultural processes as possibility of modeling and designing of social interaction. Actual problems of socio-cultural and art design, Moscow: Rhythm, 8, $51-70$.

Kamenets, A., \& Seleznyova, E. (2013). The synergetic analysis of socio-cultural transformations of the Russian society in innovative strategies of the XXI century. Actual problems of socio-cultural and art design, Moscow: Perspective, 9, 67-87.

Kamenets, A., \& Urmina, I. (2011). Technologies of social interaction in the solution of actual problems of youth. Moscow: RSSU.

Kenneth, M., Boykinb, A., \& Tia, R. (2006). Cultural considerations in teachers' perceptions of student classroom behavior and achievement. Waltonb Teaching and Teacher Education, 22(8), 998-1005. http://dx.doi.org/10.1016/j.tate.2006.04.017

Kholopov, Yu. (2008). Introduction to a musical form. Moscow: Moscow Conservatory.

Nakahashi, W. (2014). The effect of cultural interaction on cumulative cultural evolution. Journal of Theoretical Biology, 352, 6-15. http://dx.doi.org/10.1016/j.jtbi.2014.02.032

Nigmatov, Z. (2014). Humanitarian Technologies of Inclusive Education. Procedia-Social and Behavioral Sciences, 131, 156-159. http://dx.doi.org/10.1016/j.sbspro.2014.04.096

Schedrovitsky, G. (1995). Selected works. Moscow: School of Cultural Policy.

Scherbakova, A. (2012). Philosophy of musical art. Moscow: RSSU.

Scherbakova, A. (2014). Philosophical comprehension of music as methodological basis of researches in the field of musical art and education. Life Sci. J., 11(11), 429-432. Retrieved September 9, 2014, from http://www.lifesciencesite.com/lsj//life1111/073_25760life111114_429_432.pdf 
Thompson, P. (2013). Learner-centred education and "cultural translation". International Journal of Educational Development, 33(1), 48-58. http://dx.doi.org/10.1016/j.ijedudev.2012.02.009

Villodre, M. B. (2014). Cultural Identity and Using Music in the Intercultural Educational Process Procedia. 6th International Conference on Intercultural Education "Education and Health: From a transcultural perspective”, Guadalupe 30107 (Spain), 235-240.

Wright, P., \& Lundy, M. (2014). Perspectives of Cultural Competency from an International Service. Learning Project Journal of the Academy of Nutrition and Dietetics, 114(7), 996-997, 999-1000.

Zeynep, B., \& Sukhvinder, S. (2014). Cultural background influences implicit but not explicit sense of agency for the production of musical tones. Consciousness and Cognition, 28, 94-103. http://dx.doi.org/10. 1016/j.concog.2014.06.013

Zinchenko, E. (2014). Specifics of socio-cultural design in leisure sphere. Human Capital, 5(65), 106-110.

\section{Copyrights}

Copyright for this article is retained by the author(s), with first publication rights granted to the journal.

This is an open-access article distributed under the terms and conditions of the Creative Commons Attribution license (http://creativecommons.org/licenses/by/3.0/). 